\title{
Fatigue modeling using neural networks: a comprehensive review
}

\author{
Jie Chen ${ }^{1}$ and Yongming Liu $^{1}$ \\ ${ }^{1}$ Arizona State University School for Engineering of Matter Transport and Energy
}

September 25, 2021

\begin{abstract}
Neural network (NN) models have made a significant impact on fatigue-related engineering communities and are expected to increase rapidly soon due to the recent advancements in machine learning and artificial intelligence. A comprehensive review of fatigue modeling methods using NNs is lacking and will help to recognize past achievements and suggest future research directions. Thus, this paper presents a survey of 251 publications between 1990 and July 2021. The NN modeling in fatigue is classified into five applications: fatigue life prediction, fatigue crack, fatigue damage diagnosis, fatigue strength, and fatigue load. A wide range of $\mathrm{NN}$ architectures are employed in the literature and are summarized in this review. An overview of important considerations and current limitations for the application of NNs in fatigue is provided. Statistical analysis for the past and the current trend is provided with representative examples. Existing gaps and future research directions are also presented based on the reviewed articles.
\end{abstract}

\section{Hosted file}

Manuscript draft.docx available at https://authorea.com/users/436705/articles/538843-fatiguemodeling-using-neural-networks-a-comprehensive-review 